\title{
Optic nerve sheath meningioma: current diagnosis and treatment
}

\author{
Christopher S. Eddleman, M.D., Ph.D., ANd James K. Liu, M.D. \\ Department of Neurological Surgery, Feinberg School of Medicine, Northwestern University, \\ Chicago; and Evanston Northwestern Healthcare, Evanston, Illinois
}

\begin{abstract}
$\checkmark$ Optic nerve sheath meningiomas (ONSMs) are rare tumors of the anterior visual pathway and constitute approximately $2 \%$ of all orbital tumors and 1-2\% of all meningiomas. Untreated ONSMs almost always lead to progressive visual decline, color blindness, and finally complete loss of vision. Although resection is warranted in cases of widespread ONSM, surgery can lead to significant morbidity. Recently, stereotactic fractionated radiotherapy has shown effectiveness in improving or stabilizing remaining visual function with minimal procedural morbidity in patients with ONSM. The authors review the incidence, histopathological characteristics, clinical presentation, neuroimaging findings, and current treatment modalities for ONSMs, with an emphasis on fractionated stereotactic radiotherapy.

(DOI: 10.3171/FOC-07/11/E4)
\end{abstract}

\section{KEY WORDS - optic nerve sheath meningioma • orbital tumor - radiosurgery • stereotactic fractionated radiation therapy • visual impairment}

$\mathrm{O}$ PTIC nerve sheath meningiomas are rare tumors of the anterior visual pathway. ${ }^{9}$ Without treatment, continued growth of ONSMs most often results in progressive visual acuity decline, color blindness, and finally complete loss of vision. . $^{1,5,7,15,15,26,28,30,34,37,38}$ Definitive treatment of ONSMs is challenging, however, because of the lesions' intimate circumferential relationship with the optic nerve and its vascular supply. Surgical excision has almost always resulted in postoperative blindness in the affected eye. ${ }^{15,7,9}$ Conservative measures may maintain some form of functional vision but almost always lead to eventual deterioration of vision. . $^{1,7,7}$ Radiotherapy, both conventional and stereotactic, has recently been shown to have good efficacy, with local tumor control and stabilization or improvement in visual function, and has been recommended as the best option for most cases of progressive or advanced disease. ${ }^{2-7,9,10,13,16,18,19,21-25,29,31,32,35}$ Despite the positive results reported in the literature, concerns regarding complications secondary to radiation-induced effects have limited the overall acceptance of radiotherapy. The increased usage of neuroimaging for nonspecific symptoms has resulted in increased detection of ONSM at earli-

Abbreviations used in this paper: $\mathrm{CT}=$ computed tomography; $\mathrm{MR}=$ magnetic resonance; ONSM = optic nerve sheath meningioma; SFRT = stereotactic fractionated radiotherapy; WHO = World Health Organization. er stages, but the optimal treatment of these ONSMs has remained poorly defined. In this article, we review the incidence, histopathological characteristics, clinical presentation, and neuroimaging findings associated with ONSM as well as current treatment modalities for these lesions, with an emphasis on fractionated stereotactic radiotherapy.

\section{Incidence and Epidemiology}

Optic nerve sheath meningiomas represent approximately $2 \%$ of all orbital tumors, as reported in large orbital tumor series by Wright et al. and Shields et al. ${ }^{7,9,28,32,37,38}$ These lesions can arise primarily from the intraorbital or intracanalicular portion of the optic nerve sheath, or they can arise from surrounding tissues, most commonly outside of the orbit, and secondarily involve the intracanalicular or intraorbital optic nerve sheath. In a review of 5000 meningiomas involving the orbit, Dutton ${ }^{9}$ reported that $92 \%$ of primary ONSMs arise from within the intraorbital nerve sheath, while only $8 \%$ are intracanalicular in origin. Only $5 \%$ of ONSMs present bilaterally, and $65 \%$ of these bilateral lesions are intracanalicular. ${ }^{7,9}$ About half of the patients who present with bilateral ONSMs also have tumors along the planum sphenoidale in continuity with these lesions, a finding that raises questions about the true origin of bilateral ONSMs. Bilateral and multifocal presentations of ONSMs are most commonly found in patients with neurofibromatosis type $2 ., 7,9$ 


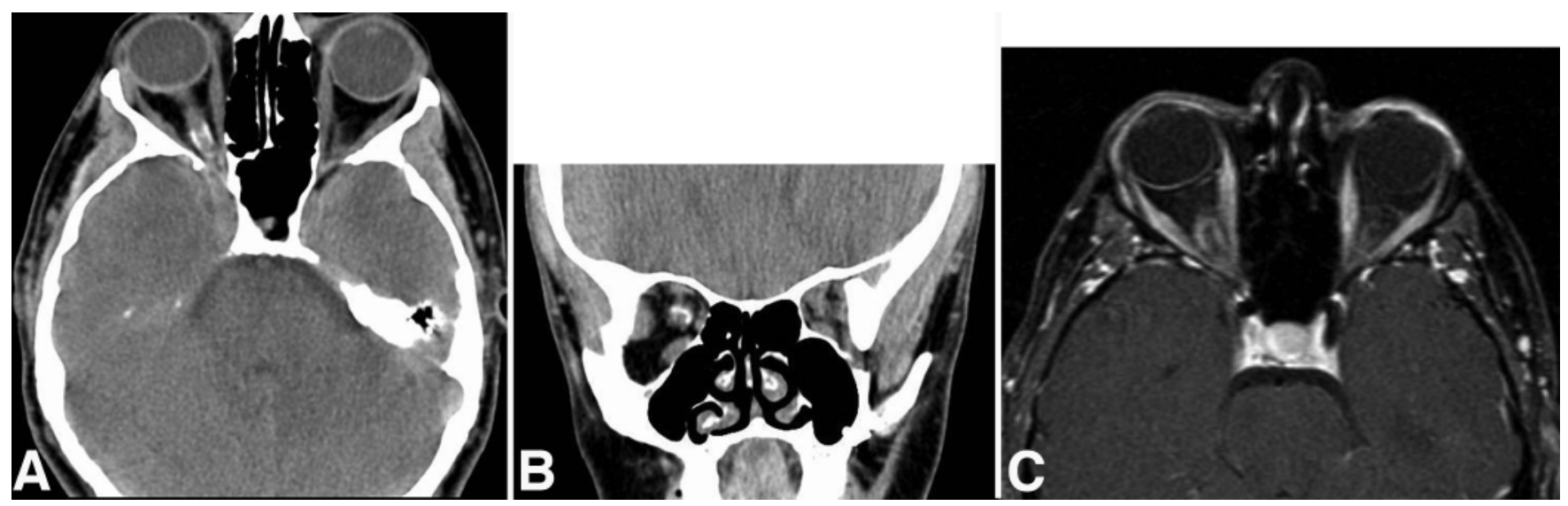

FIG. 1. Computed tomography (A and B) and MR (C) images. Axial CT (A) and coronal reconstruction (B) demonstrating circumferential calcification around the optic nerve in a patient with ONSM. C: A T1-weighted fat-suppressed Gd-enhanced MR image showing the ONSM encasing the right optic nerve. "Tram tracking" can be seen on the CT images as well as the MR image.

In a review of the ONSM literature, Dutton ${ }^{9}$ reported the mean age at presentation of ONSM patients to be 40.8 years (42.5 years in women and 36.1 years in men; range 3-80 years), with $61 \%$ of the patient population being female, a degree of female predominance similar to that associated with intracranial meningioma. Pregnancy may also exacerbate the growth of ONSMs. Approximately 4-7\% of ONSMs occur in patients younger than ${ }^{20}$ years of age. Unlike adult ONSM patients, there is no gender preference in children with ONSMs, and in the pediatric population the lesions are often associated with neurofibromatosis type 2.5, Furthermore, ONSMs in pediatric patients are frequently more aggressive, are associated with higher rates of intracranial extension on presentation, have a higher rate of bilateral presentation, and have higher rates of recurrence. ${ }^{5,79}$

\section{Pathobiology and Histopathology}

Primary ONSMs arise from meningoepithelial cap cells of the arachnoid villi and can develop at any location along the entire course of the optic nerve sheath. ., $^{7,14,26}$ Secondary ONSMs may arise from tissues outside the orbit, namely the cavernous sinus, falciform ligament, clinoid processes, sphenoid wing, pituitary fossa, planum sphenoidale, tuberculum sellae, frontotemporal dura, and/or olfactory groove, and secondarily grow into the optic nerve sheath. The growth pattern is typical for meningiomas, and growth normally follows the path of least resistance. True ONSMs arise from the area between the arachnoid and the dural sheaths of the optic nerve; secondary ONSMs arise from the intracranial cavity and grow into this space.

Although rare, invasion of the surrounding dura, orbital tissue, muscle, and bone has been reported. As ONSMs progress, it is thought that they compromise optic nerve function mainly by mass effect on the pial vascular supply, which induces ischemic changes as well as interfering with axonal transport. $714,15,34,37,38$ Furthermore, ONSMs tend to grow circumferentially around the optic nerve, interposed between the nerve substance and its blood supply, thus rendering most ONSMs unresectable without significant optic nerve compromise. ${ }^{5,7,34}$
ONSMs usually present with either of two histological patterns, namely a meningothelial or syncytial pattern, in which polygonal cells are arranged in sheets separated by vascular trabeculae, or a transitional pattern, in which spindle or oval cells are arranged in whorls, often resulting in centralized hyalinization and deposition of calcium salts (psammoma bodies). 5,7,34 As with intracranial meningiomas, the WHO classification identifies 3 histological grades of ONSMs: I, benign; II, atypical; and III, malignant. Recurrence rates are associated with WHO malignancy grade and have been reported to be $6.9,34.6$, and $72.7 \%$, respectively. $5,7,34$

\section{Clinical Presentation}

The classic triad of optic atrophy, visual loss, and the presence of opticociliary shunt vessels is pathognomonic for the clinical presentation of ONSMs; however, this is present in only a minority of patients. , $, 7,22,30^{\text {The most com- }}$ mon presenting symptom in patients with ONSMs (present in approximately $96 \%$ of cases) is painless, progressive loss of visual acuity or visual field, with progression often lasting 1-5 years before presentation., $5,22,30$ In Dutton's review of ONSMs, ${ }^{9} 45 \%$ of patients with ONSM presented with vision of $20 / 40$ or better, whereas $25 \%$ had extremely poor vision (could only count fingers, minimal light perception, or no light perception). Other associated symptoms that can occur before, during, or following loss of visual acuity or visual field in patients with ONSMs are dyschromatopsia, afferent pupillary defects, scotomas, altitudinal defects, and spontaneous or gaze-evoked transient visual obscurations, which are almost always associated with swelling of the optic disc. ${ }^{7,30}$ Funduscopic examination almost always demonstrates a pathological appearance of the optic disc, which may consist of optic disc edema with varying degrees of optic atrophy, suggesting some manifestation of a compressive optic neuropathy. Other common findings in cases of ONSM include mild to moderate proptosis, chemosis, lid edema, and limitation of extraocular muscle excursion. ${ }^{7,30}$ Other nonspecific complaints may include orbital pain and headaches, which have been reported in $2-50 \%$ of patients with ONSM. ${ }^{5,722,30}$ 

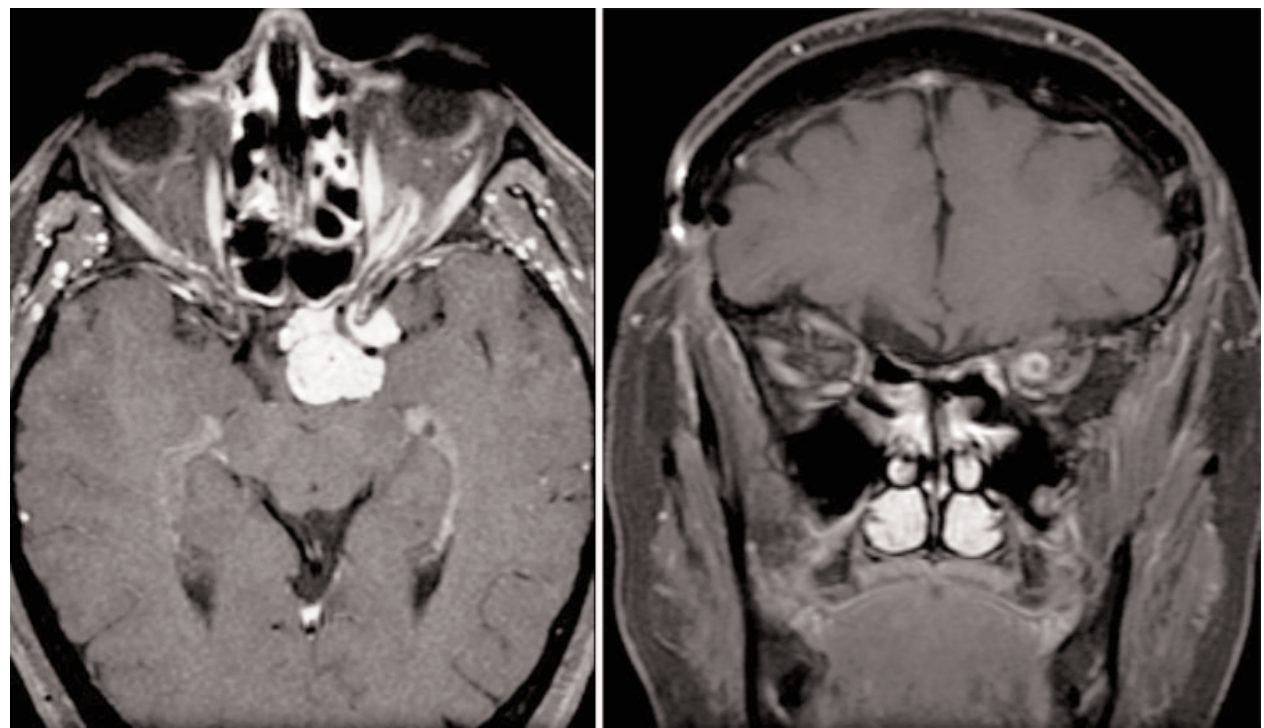

FIG. 2. Axial (left) and coronal (right) T1-weighted fat-suppressed Gd-enhanced MR images showing a suprasellar cavernous sinus meningioma with secondary involvement of the left optic nerve sheath. The "tram tracking" sign is seen on the axial view (left) and the "doughnut" sign is seen on the coronal view (right).

\section{Imaging Characteristics}

Magnetic resonance imaging has become the gold standard for visualization of ONSMs (Figs. 1-3). ${ }^{20,34}$ Although $\mathrm{CT}$ allows visualization of the tumor, only MR imaging (especially with post-gadolinium fat-suppression sequences) can significantly delineate the optic nerve as it courses through the optic canal. ${ }^{20,34}$ Advances in neuroimaging have allowed the diagnosis of ONSM to be made on the basis of clinical and neuroimaging findings, thus obviating the need for a tissue biopsy. ${ }^{1,9,11,14,20}$ Early detection of ONSM is essential to initiate early intervention and prevent subsequent growth into the intracranial cavity.

Like intracranial meningiomas, ONSMs are contrastenhancing lesions. The classic imaging "tram-tracking" sign can be easily visualized and consists of the thickened optic nerve sheath containing the lesion surrounding the nonenhancing, radiolucent optic nerve. ${ }^{20,34}$ This thickened optic nerve can appear as a calcified nerve sheath on CT images (Fig. 1). On coronal views, the tumor demonstrates a doughnut shape, with the dense nerve sheath tumor encircling the optic nerve (Figs. 2 and 3). Several imaging patterns of ONSM growth have been observed, namely tubular (diffuse, apical expansion, anterior expansion), globular, fusiform, and focal. ${ }^{7,20,34}$ The most common tubular form represents the classic circumferential morphological pattern extending either to the orbital apex or the globe or simply focal, diffuse expansion. Globular patterns represent exophytic expansion beyond the optic nerve sheath. Finally, lesions that have a fusiform growth pattern appear elliptical, with tapered ends, and are often mistaken for optic gliomas. Some lesions are completely exophytic and probably represent extradural growth without direct involvement of the optic nerve sheath. Such lesions are likely to be orbital meningiomas not originating from within the optic nerve sheath, and thus would not be true ONSMs. These specific lesions are frequently successfully treated with resection without visual compromise due to their sep- aration from the optic nerve vascular supply, unlike true ONSMs, which, as a rule, cannot be treated surgically without loss. Orbital ultrasonography has also been used, especially by ophthalmologists, and relies mainly on the often consistent diameter of the optic nerve sheath. An increase in nerve sheath diameter typically warrants a more detailed imaging study, which can often lead to the diagnosis of ONSM. ${ }^{34}$

\section{Management and Outcome}

The goal of treatment for patients with ONSMs is tumor control and preservation or improvement of vision. With respect to prognosis, ONSMs are essentially associated with a mortality rate of $0 \%,,^{5,9}$ but untreated, the lesions almost always result in progressive visual deterioration. Therefore, treatment of ONSM has, as a rule, been considered except in cases in which the progression of visual loss is negligible or other medical issues prevent the recommended therapy.

\section{Observation Without Treatment}

Historically, management of ONSMs has involved either observation without treatment, mostly in cases of high functional vision or negligible visual decline, or resection, especially in cases of threatening intracranial extension or disfiguring proptosis. Observation may be warranted in some cases, as mentioned above, but $85 \%$ of patients experience visual decline, as described by Kennerdell et al. ${ }^{14}$ and Turbin et al..$^{35}$ Furthermore, in cases of intracanalicular ONSMs, lesion-associated effects in the contralateral eye have been reported in up to $38 \%$ of cases. Thus there is a substantial risk that both eyes may be affected in the absence of treatment. ${ }^{5,9}$ Moreover, allowing progression of ONSM growth and consequent visual deterioration may decrease the opportunity for visual improvement after treatment. ${ }^{5}$ In pediatric patients, ONSMs frequently exhibit 

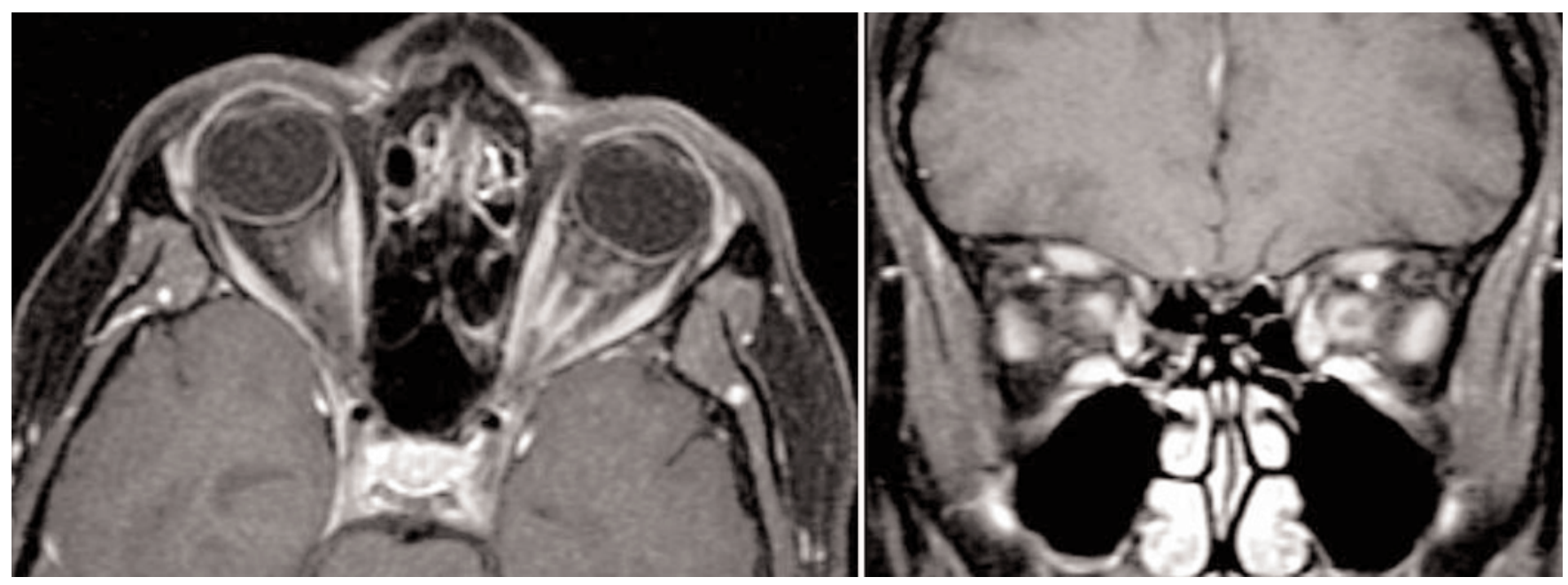

FIG. 3. Axial (left) and coronal (right) T1-weighted fat-suppressed Gd-enhanced MR images showing bilateral ONSMs.

more aggressive behavior, and young patients are at increased risk for progressive visual decline. Thus, recommendations for observation without treatment should be followed only with caution.

While observation may be warranted in certain cases, especially those in which visual acuity of 20/40 or better has been maintained, close follow-up, with frequent clinical examinations, including assessment of visual acuity, color vision, and visual fields, should be performed biannually for at least 2-3 years, after which they can be performed annually if visual function has stabilized. ${ }^{5,13}$ Imaging evaluation should also be performed at 6-month intervals for 1-2 years and then annually for several more years to establish stable ONSM appearance. Shorter intervals between follow-up examinations are recommended for younger patients.

\section{Surgical Treatment}

Before detailed imaging studies became available, surgical biopsy was often performed, especially when the clinical presentation was atypical. However, obtaining an adequate amount of tissue for diagnosis often required extensive exposures, which subjected patients to subsequent procedural complications. ${ }^{5,7,15,22,27}$ Furthermore, the diagnosis of ONSM can be mistaken due to the presence of meningeal hyperplasia, which is often seen in cases of optic glioma. ${ }^{7}$ Finally, surgical biopsies of ONSMs are associated with high visual morbidities. 5,7,22 Thus, ONSM patients with functional vision were subjected to a procedure that frequently led to complications and often did not lead to a useful diagnosis; therefore, surgical biopsies have been largely abandoned, Currently, biopsies are considered unnecessary because of the characteristic clinical presentation and examination findings and advanced neuroimaging techniques.

Another attempt at surgical treatment without resection of ONSMs involved fenestrating the optic nerve sheath., ${ }^{15,34}$ Opening the dural sheath was thought to relieve compression of the optic nerve, thus allowing the possibility of improved visual function. Despite some successful cases being reported, in the majority of patients with ONSM who underwent this treatment, visual function did not improve and even declined due to subsequent massive orbital invasion. Furthermore, any improvement that was observed was frequently temporary due to the continued progression of tumor growth. For these reasons optic nerve sheath fenestration is rarely performed.

Complete resection of the optic nerve was first described as a primary therapy for ONSM in 1938 by Cushing and Eisenhardt. ${ }^{8}$ Despite the subsequent common practice of resection of ONSMs, the ability to preserve visual function after such excisions is extremely rare., ${ }^{5,15}$ Primary ONSMs usually wrap circumferentially around the optic nerve, and their removal cannot be successfully completed without compromising the integrity of the vascular supply of the optic nerve.

Although resection almost always leads to compromised visual function, there are cases of ONSMs in which resection may be warranted, namely in patients whose functional vision is already significantly compromised, in patients who have disfiguring orbital proptosis, and in patients in whom intracranial extension is present and growing toward the contralateral optic nerve. ${ }^{9,14}$ In patients who have no visual function in the affected eye, en bloc resection of the tumor and involved optic nerve can be performed. If the globe is invaded by tumor and orbital proptosis is present, orbital exenteration should be considered. In patients with rapidly deteriorating vision, surgery for the goal of immediate optic decompression may be considered, especially if there is tumor within and around the optic canal with intracranial extension. This type of operation would include a frontotemporal craniotomy, posterior orbitotomy, anterior clinoidectomy, optic roof decompression, and opening of the falciform ligament and dura propria of the optic nerve. ${ }^{27}$ If there is intracranial meningioma present, such as a clinoidal or tuberculum sellae meningioma, special consideration should be taken to remove the intracranial portion in order to prevent spread to the normal contralateral optic nerve, thus sparing vision in the good eye.

Visual improvement after ONSM resection has been reported in the literature, albeit rarely, and usually in associ- 
TABLE 1

Summary of studies of SFRT for the treatment of ONSM*

\begin{tabular}{|c|c|c|c|c|c|c|c|c|c|}
\hline \multirow[b]{2}{*}{ Authors \& Year } & \multirow{2}{*}{$\begin{array}{c}\text { No. of Eyes } \\
\text { Treated }\end{array}$} & \multirow[b]{2}{*}{ Study Period } & \multirow{2}{*}{$\begin{array}{l}\text { Treatment } \\
\text { Mode }\end{array}$} & \multirow{2}{*}{$\begin{array}{l}\text { Max Total } \\
\text { Dose (Gy) }\end{array}$} & \multirow{2}{*}{$\begin{array}{l}\text { Fraction } \\
\text { (Gy) }\end{array}$} & \multicolumn{3}{|c|}{ Outcome (no. of eyes) } & \multirow{2}{*}{$\begin{array}{l}\text { Complications } \\
\text { (no. of cases) }\end{array}$} \\
\hline & & & & & & Improved & Stable & Worse & \\
\hline Andrews et al., 2002 & 24 & 1996-2001 & conformal SFRT & 54 & 1.8 & 10 & 12 & 2 & optic neuritis (1) \\
\hline Liu et al., 2002 & 5 & 1994-2001 & SFRT & 54 & 1.8 & 4 & 1 & 0 & none \\
\hline Pitz et al., 2002 & 15 & 1989-2000 & conformal SFRT & 54 & 3.6 & 6 & 3 & 6 & pituitary abnormality (2) \\
\hline Saeed et al., 2003 & 6 & 1976-1999 & conformal SFRT & 45 & NR & 5 & 0 & 1 & cataract (1) \\
\hline Baumert et al., 2004 & 23 & 1996-2003 & conformal SFRT & 60 & $1.8-2.0$ & 16 & 5 & 2 & vitreous hemorrhage (1) \\
\hline Richards et al., 2005 & 4 & 1999-2002 & SFRT & 47.25 & $1.7-1.75$ & 4 & 0 & 0 & retinopathy (1) \\
\hline Sitathanee et al., 2006 & 12 & 1998-2005 & SFRT & 55.7 & 1.8 & 4 & 7 & 1 & vitreous hemorrhage (1) \\
\hline
\end{tabular}

$* \mathrm{NR}=$ not reported

ation with ONSMs that were small, situated anteriorly, and close to the globe, and were not associated with significant neural invasion. In Dutton's review of ONSMs treated with resection, ${ }^{9}$ the mortality rate was $0 \%$, the rate of operative complication was $30 \%$, and the recurrence rate was $25 \%$. Visual outcomes after resection showed improvement in only $5 \%$ of cases, with most patients $(78 \%)$ experiencing no light perception.

\section{Radiotherapy With or Without Resection}

In an attempt to preserve visual function, subtotal resection with postoperative radiotherapy became a highly utilized strategy for combined therapy. Despite the historical notion that meningiomas in general were considered radioresistant, visual stabilization or even improvement was reported by several groups..$^{5,7,12,13,15}$ Nevertheless, postoperative complication rates did not differ significantly in patients treated with combined therapy as compared with surgery alone. Given that visual improvement had been demonstrated with combined therapy, several investigators examined the use of conventional radiotherapy in lieu of surgery. Smith et al. ${ }^{32}$ were the first authors to clearly demonstrate the efficacy of radiotherapy in patients with primary ONSMs. Dutton ${ }^{9}$ examined the literature with respect to ONSMs treated by means of radiotherapy and reported that visual acuity improved in $75 \%$ of cases, remained stable in $8 \%$, and declined in $17 \%$. Widespread use of radiotherapy for the treatment ONSMs did not flourish at that time, primarily due to fears of radiation toxicity, even though complication rates at the doses used in those studies (approximately 54 Gy given in daily 1.8-Gy fractions) were less than $2 \%$.

Turbin et al. ${ }^{35}$ reported on 64 patients with ONSMs in whom the lesions had been managed with either observation alone, surgery alone, surgery with postoperative radiotherapy, or radiotherapy alone and concluded that treatment with radiotherapy alone resulted in the best long-term visual outcomes. Nevertheless, $33 \%$ of these patients developed complications from conventional radiotherapy, including radiation retinopathy, retinal vascular occlusion, persistent iritis, and temporal lobe atrophy. These complications were attributed to nonconformal delivery of radiation.
Technological development—namely the ability to administer conformal radiation doses to a tumor, thereby reducing adjacent tissue exposure which would theoretically minimize radiation toxicity-led to increased primary treatment of ONSMs using radiotherapy alone. Several different techniques, namely stereotactic fractionated radiosurgery, intensity-modulated radiotherapy, SFRT, and 3Dconformal radiotherapy, have shown promising results, with stable or improved vision at follow-up times of up to 2 years. $^{2-5,7,9,10,13,16-19,21-25,29,31-33,35,36}$

Using SFRT it is possible to deliver a sufficient dose of radiation to an ONSM in a more focused, conformal beam, thereby sparing surrounding tissues from high radiation doses and minimizing radiation-induced complications (Table 1). Pitz et al. ${ }^{24}$ reported improvement of visual acuity or visual fields in $58 \%$ of patients with ONSMs who were treated with stereotactic fractionated radiotherapy; none experienced visual decline. Baumert et al. ${ }^{3}$ reported on the treatment of 23 eyes with ONSMs using highly conformal SFRT with a mean follow-up period of 20 months and demonstrated visual improvement in 16 eyes $(70 \%)$ and stable vision in $5(22 \%)$. Two patients had worse vision, and in one of these two radiation retinopathy developed. Berman and Miller ${ }^{5}$ summarized the data from 7 large case series in which a total of 75 patients with ONSM were treated with SFRT; the authors reported an overall disease control of $94.6 \%$ and visual improvement in $54.7 \%$ occurring within the first 3 months after treatment. None of the patients demonstrated tumor growth in subsequent imaging studies. Complications in these series included headache, nausea, local erythema, focal alopecia, and radiation retinopathy. Other complications such as late pituitary dysfunction and cerebral small vessel disease have been reported in up to $10 \%$ of patients treated with SFRT. ${ }^{13}$ As more experience with SFRT has been reported, improvements in dose administration and planning have led to better ONSM treatment results. Richards et al. ${ }^{25}$ treated ONSM with a slightly lower prescribed dose (total dose of 43.4 to 45 Gy, daily 1.67 Gy doses), and achieved improvement of visual acuity and visual fields in $65 \%$ of patients. Landert et al. ${ }^{18}$ used doses of 50.4 to 54 Gy and demonstrated visual acuity improvement in $86 \%$ and visual field improvement in $57 \%$ of patients. No complications were 
noted in either study. Finally, Sitathanee et al. ${ }^{31}$ reported on the SFRT treatment of 12 patients with ONSM, 5 of whom presented with no light perception and had undergone previous surgery, and demonstrated no improvement in the patients who presented with no light perception but showed visual improvement in 4 and stable vision in 2 patients who still had functioning vision. One complication-vitreous hemorrhage-occurred 2 years after treatment.

\section{Conclusions}

Stereotactic fractionated radiotherapy has been shown to offer the most consistent and effective treatment for ONSMs, resulting in preservation and improvement in visual function in approximately $80 \%$ of patients. As technology continues to develop, improvement in planning and targeting strategies of SFRT for the treatment of ONSMs will probably lead to improvement in visual outcomes, thus giving patients suffering from this benign yet potentially vision-threatening tumor an increased chance of maintaining visual function.

\section{References}

1. Alper MG: Management of primary optic nerve meningiomas. Current status - therapy in controversy. J Clin Neuroophthalmol 1:101-117, 1981

2. Andrews DW, Faroozan R, Yang BP, Hudes RS, Werner-Wasik M, Kim SM, et al: Fractionated stereotactic radiotherapy for the treatment of optic nerve sheath meningiomas: preliminary observations of 33 optic nerves in 30 patients with historical comparison to observation with or without prior surgery. Neurosurgery 51:890-904, 2002

3. Baumert BG, Villa S, Studer G, Mirimanoff RO, Davis JB, Landau K, et al: Early improvements in vision after fractionated stereotactic radiotherapy for primary optic nerve sheath meningioma. Radiother Oncol 72:169-174, 2004

4. Becker G, Jeremic B, Pitz S, Buchgeister M, Wilhelm H, Schiefer $\mathrm{U}$, et al: Stereotactic fractionated radiotherapy in patients with optic nerve sheath meningioma. Int J Radiat Oncol Biol Phys 54:1422-1429, 2002

5. Berman D, Miller NR: New concepts in the management of optic nerve sheath meningiomas. Ann Acad Med Singapore 35: 168-174, 2006

6. Capo H, Kupersmith MJ: Efficacy and complications of radiotherapy of anterior visual pathway tumors. Neurol Clin 9: 179-203, 1991

7. Carrasco JR, Penne RB: Optic nerve sheath meningiomas and advanced treatment options. Curr Opin Ophthalmol 15: 406-410, 2004

8. Cushing H, Eisenhardt L: Meningiomas. Their Classification, Regional Behavior, Life History and Surgical End Results. Springfield, Ill: Charles C. Thomas, 1938, pp 283-297

9. Dutton JJ: Optic nerve sheath meningiomas. Surv Ophthalmol 37:167-183, 1992

10. Eng TY, Albright NW, Kuwahara G, Akazawa CN, Dea D, Chu GL, et al: Precision radiation therapy for optic nerve sheath meningiomas. Int J Radiat Oncol Biol Phys 22:1093-1098, 1992

11. Fineman MS, Augsburger JJ: A new approach to an old problem. Surv Ophthalmol 43:519-524, 1999

12. Ito M, Ishizawa A, Miyaoka M, Sato K, Ishii S: Intraorbital meningiomas. Surgical management and role of radiation therapy. Surg Neurol 29:448-453, 1988

13. Jeremic B, Pitz S: Primary optic nerve sheath meningioma: stereo- tactic fractionated radiation therapy as an emerging treatment of choice. Cancer 110:714-722, 2007

14. Kennerdell JS, Maroon JC, Malton M, Warren FA: The management of optic nerve sheath meningiomas. Am J Ophthalmol 106:450-457, 1988

15. Kim JW, Rizzo JF, Lessell S: Controversies in the management of optic nerve sheath meningiomas. Int Ophthalmol Clin 45: 15-23, 2005

16. Klink DF, Miller NR, Williams J: Preservation of residual vision 2 years after stereotactic radiosurgery for a presumed optic nerve sheath meningioma. J Neuroophthalmol 18: 117-120, 1998

17. Kondziolka D, Lunsford LD, Coffey RJ, Flickinger JC: Stereotactic radiosurgery of meningiomas. J Neurosurg 74:552-559, 1991

18. Landert M, Baumert BG, Bosch MM, Lutolf UM, Landau K: The visual impact of fractionated stereotactic conformal radiotherapy on seven eyes with optic nerve sheath meningiomas. J Neuroophthalmol 25:86-91, 2005

19. Lee AG, Woo SY, Miller NR, Safran AB, Grant WH, Butler EB: Improvement in visual function in an eye with a presumed optic nerve sheath meningioma after treatment with three-dimensional conformal radiation therapy. J Neuroophthalmol 16:247-251, 1996

20. Lindblom B, Truwit CL, Hoyt WF: Optic nerve sheath meningioma. Definition of intraorbital, intracanalicular, and intracranial components with magnetic resonance imaging. Ophthalmology 99:560-566, 1992

21. Liu JK, Forman S, Hershewe GL, Moorthy CR, Benzil DL: Optic nerve sheath meningiomas: visual improvement after stereotactic radiotherapy. Neurosurgery 50:950-957, 2002

22. Liu JK, Forman S, Moorthy CR, Benzil DL: Update on treatment modalities for optic nerve sheath meningiomas. Neurosurg Focus 14: E7, 2003

23. Moyer PD, Golnik KC, Breneman J: Treatment of optic nerve sheath meningioma with three-dimensional conformal radiation. Am J Ophthalmol 129:694-696, 2000

24. Pitz S, Becker G, Schiefer U, Wilhelm H, Jeremic B, Bamberg M, et al: Stereotactic fractionated irradiation of optic nerve sheath meningioma: a new treatment alternative. Br J Ophthalmol 86: 1265-1268, 2002

25. Richards JC, Roden D, Harper CS: Management of slight-threatening optic nerve sheath meningioma with fractionated stereotactic radiotherapy. Clin Experiment Ophthalmol 33:137-141, 2005

26. Saeed P, Rootman J, Nugent RA, White VA Mackenzie IR, Koornneef L: Optic nerve sheath meningiomas. Ophthalmology 110:2019-2030, 2003

27. Schick U, Dott U, Hassler W: Surgical management of meningiomas involving the optic nerve sheath. J Neurosurg 101: 951-959, 2004

28. Shields JA, Shields CL, Scartozzi R: Survey of 1264 patients with orbital tumors and simulating lesions. The 2002 Montgomery Lecture, part 1. Ophthalmology 111:997-1008, 2004

29. Shrieve DC, Tarbell NJ, Alexander E III, Kooy HM, Black PM, Dunbar S, et al: Stereotactic radiotherapy: a technique for dose optimization and escalation for intracranial tumors. Acta Neurochir Suppl 62:118-123, 1994

30. Sibony PA, Krauss HR, Kennerdell JS, Maroon JC, Slamovits TL: Optic nerve sheath meningiomas. Clinical manifestations. Ophthalmology 91:1313-1326, 1984

31. Sitathanee C, Dhanachai M, Poonyathalang A, Tuntiyatorn L, Theerapancharoen V: Stereotactic radiation therapy for optic nerve sheath meningioma: an experience at Ramathibodi Hospital. J Med Assoc Thai 89:1665-1669, 2006

32. Smith JL, Vuksanovic MM, Yates BM, Bienfang DC: Radiation therapy for primary optic nerve meningiomas. J Clin Neuroophthalmol 1:85-99, 1981

33. Tishler RB, Loeffler JS, Lunsford LD, Duma C, Alexander E III, Kooy HM, et al: Tolerance of cranial nerves of the cavernous 
sinus to radiosurgery. Int J Radiat Oncol Biol Phys 27:215-221, 1993

34. Turbin RE, Pokorny K: Diagnosis and treatment of orbital optic nerve sheath meningioma. Cancer Control 11:334-341, 2004

35. Turbin RE, Thompson CR, Kennerdell JS, Cockerham KP, Kupersmith MJ: A long-term visual outcome comparison in patients with optic nerve sheath meningioma managed with observation, surgery, radiotherapy, or surgery and radiotherapy. Ophthalmology 109:890-900, 2002

36. Wara WM, Sheline GE, Newman H, Townsend JJ, Boldrey EB: Radiation therapy of meningiomas. AJR Radium Ther Nucl Med 123:453-458, 1975
37. Wright JE, Call NB, Liaricos S: Primary optic nerve meningioma. Br J Ophthalmol 64:553-558, 1980

38. Wright JE, McNab AA, McDonald WI: Primary optic nerve sheath meningioma. Br J Ophthalmol 73:960-966, 1989

Manuscript submitted August 15, 2007.

Accepted September 14, 2007.

Address correspondence to: James K. Liu, M.D., Evanston Northwestern Healthcare, Department of Neurological Surgery, 2650 Ridge Avenue, Evanston, Illinois 60201. email: jliu@enh.org. 